\title{
Accelerated cell cycle progression of human regulatory T cell-like cell line caused by continuous exposure to asbestos fibers
}

\author{
SUNI LEE ${ }^{1}$, HIDENORI MATSUZAKI ${ }^{1}$, MEGUMI MAEDA ${ }^{2}$, SHOKO YAMAMOTO $^{1}$, \\ NAOKO KUMAGAI-TAKEI ${ }^{1}$, TAMAYO HATAYAMA ${ }^{1}$, MIHO IKEDA ${ }^{1}$, \\ KEI YOSHITOME ${ }^{1}$, YASUMITSU NISHIMURA ${ }^{1}$ and TAKEMI OTSUKI ${ }^{1}$ \\ ${ }^{1}$ Department of Hygiene, Kawasaki Medical School, Kurashiki, Okayama 701-0192; \\ ${ }^{2}$ Department of Biofunctional Chemistry, Division of Bioscience, Okayama University Graduate School \\ of Natural Science and Technology, Kita-Ku, Okayama 700-8530, Japan
}

Received September 21, 2016; Accepted November 18, 2016

DOI: 10.3892/ijo.2016.3776

\begin{abstract}
Asbestos exposure causes malignant tumors such as lung cancer and malignant mesothelioma. Based on our hypothesis in which continuous exposure to asbestos of immune cells cause reduction of antitumor immunity, the decrease of natural killer cell killing activity with reduction of NKp46 activating receptor expression, inhibition of cytotoxic T cell clonal expansion, reduced CXCR3 chemokine receptor expression and production of interferon- $\gamma$ production in $\mathrm{CD}^{+} \mathrm{T}$ cells were reported using cell line models, freshly isolated peripheral blood immune cells from health donors as well as asbestos exposed patients such as pleural plaque and mesothelioma. In addition to these findings, regulatory $\mathrm{T}$ cells (Treg) showed enhanced function through cell-cell contact and increased secretion of typical soluble factors, interleukin (IL)-10 and transforming growth factor (TGF)- $\beta$, in a cell line model using the MT-2 human polyclonal T cells and its sublines exposed continuously to asbestos fibers. Since these sublines showed a remarkable reduction of FoxO1 transcription factor, which regulates various cell cycle regulators in asbestosexposed sublines, the cell cycle progression in these sublines was examined and compared with that of the original MT-2 cells. Results showed that cyclin D1 expression was markedly enhanced, and various cyclin-dependent kinase-inhibitors were reduced with increased $\mathrm{S}$ phases in the sublines. Furthermore, the increase of cyclin D1 expression was regulated by FoxO1. The overall findings indicate that antitumor immunity in asbestos-exposed individuals may be reduced in Treg through changes in the function and volume of Treg.
\end{abstract}

Correspondence to: Dr Takemi Otsuki, Department of Hygiene, Kawasaki Medical School, 577 Matsushima, Kurashiki, Okayama 701-0192, Japan

E-mail: takemi@med.kawasaki-m.ac.jp

Key words: asbestos, regulatory T cell, cell cycle, FoxO1, cyclin D1

\section{Introduction}

Asbestos exposure causes lung fibrosis known as asbestosis, one of the most typical forms of pneumoconiosis, as well as pleural plaque (PP), benign pleural effusion, and diffuse pleural thickening (1-3). In addition to these benign diseases, the occurrence of malignant tumors such as lung cancer and malignant mesothelioma (MM) is an important consideration for the understanding and development of disease prevention methods, early diagnosis and treatment (4-6). The carcinogenic potential of asbestos fibers has been recognized. Initial DNA damage caused by production of reactive oxygen species (ROS) mainly derived from iron-including asbestos such as crocidolite (CRD) and amosite is considered the dominant cause of asbestos-induced carcinogenesis $(7,8)$. Next, physiological DNA damage caused by asbestos fibers to pulmonary epithelial cells and pleural mesothelial cells is the other cause of malignant transformation because of the rigid and thin physical features of fibers with more than a 3 aspect ratio for fibers defined as a 'fiber' $(9,10)$. In addition to these causes, the easy absorbance of other carcinogenic substances inhaled into the lung, such as tobacco smoke and air pollutants on the surface of asbestos fibers, may enhance the synergistic effects for malignant transformation, especially for lung epithelial cells, since the odds ratio for lung cancer caused by asbestos inhalation and tobacco smoking showed synergistic effects according to various epidemiological studies (9-11).

We have been investigating the immunological effects of asbestos fibers because asbestos is a mineral silicate possessing $\mathrm{Si}$ and $\mathrm{O}$ as the core chemicals, and silica $\left(\mathrm{SiO}_{2}\right)$ has been shown to affect immune competent cells. The evidence of dysregulation of the immune system caused by silica was confirmed epidemiologically by frequent complications of autoimmune diseases, as well as cell biological investigations which revealed chronic activation of responder and regulatory T cells (Treg; CD4 ${ }^{+}, \mathrm{CD}_{25}{ }^{+}$and forkhead box P3 (FoxP3) positive inhibitory cells) caused by silica particles (12-14). Considering the malignant complications in asbestos-exposed patients, it is possible that asbestos exposure promotes the 
reduction of tumor immunity in these patients $(15,16)$. It is on the basis of this viewpoint that we examined the effects of asbestos on human immune cells. Investigations involving human natural killer (NK) cells, a cell line and freshly isolated NK cells derived from healthy donors (HD) and stimulated ex vivo, showed reduction of NK cell killing activity with decreased expression of some NK cell-activating receptors such as NKG2D and 2B4, with the suppression of the mitogenactivated protein kinase (MAPK) signaling pathway $(17,18)$. In addition, the most remarkable reduction among these activating receptors was NKp46 when comparing its expression to freshly isolated NK cells derived from HD, PP and MM patients $(17,18)$. The expression of NKp46 on the surface of NK cells was well correlated with NK cell killing activities in these patients. Differentiation and clonal expansion of $\mathrm{CD}^{+}$ cytotoxic T lymphocytes (CTLs) were inhibited when chrysotile asbestos was co-cultured in a mixed lymphocyte reaction (MLR) assay with reduction of cell attacking molecules such as granzyme B and perforin (19,20).

Experiments involving $\mathrm{T}$ cells have been developed using cell line models with continuous exposure to chrysotile A (ChA), chrysotile B (ChB) or CRD for more than one year in vitro, with cells derived from the human $\mathrm{T}$ cell leukemia/ lymphoma (HTLV)-1 virus immortalized human polyclonal cell line, MT-2. The original MT-2 cells (Org) showed apoptosis with activation of pro-apoptotic MAPK, mitochondrial apoptotic pathway and ROS production, when exposed transiently to $\mathrm{ChA}, \mathrm{ChB}$ or CRD with relatively high doses of these fibers (21-24). However, when Org cells (MT-2 cells that never encountered asbestos fibers) were exposed continuously with relatively low doses of fibers (doses that do not yield apoptosis in less than half of the cells) for more than one year, these sublines (designated as CB1 to 3, CA1 to 3, and CR1 exposed to $\mathrm{ChB}, \mathrm{ChA}$ and CRD, respectively, and established independently) exhibited changes in cell features $(15,22,24)$. The cells showed acquisition of asbestos-induced apoptosis, alteration of cytokine production, excess production of interleukin (IL)-10 and transforming growth factor (TGF)- $\beta$, reduced production of interferon (IFN)- $\gamma$, resistance to TGF- $\beta$-induced growth inhibition, and enhanced phosphorylation and expression of $\beta$-actin on their cell surface $(15,22-25)$. In addition to these alterations, the cell surfaces in these sublines showed reduction of C-X-C motif chemokine receptor 3 (CXCR3), which is known to be important to attract IFN- $\gamma$-producing and tumor attacking $\mathrm{T}$ cells near tumor cells (26-28). Coupled with reduced secretion of IFN- $\gamma$, these sublines were characterized by reduced antitumor immunity. These findings were confirmed in freshly isolated $\mathrm{CD}^{+} \mathrm{T}$ cells derived from patients with PP or MM (26-28). Thus, considering these results and those obtained from NK cells and CTLs indicated that asbestos exposure causes reduction of antitumor immunity in asbestos-exposed individuals.

Since it was reported that MT-2 cells possess Treg function (29) and exhibit excess production of typical soluble factors such as Treg, IL-10 and TGF- $\beta$ in CB1-3, CA1-3 and $\mathrm{CR}$ sublines continuously exposed to asbestos fibers such as $\mathrm{ChB}, \mathrm{ChA}$ and CRD (15,22-25), it was thought that asbestos exposure of the MT-2 cell line causes enhanced Treg function. This possibility was investigated together with the increase in
Treg function of sublines through cell cell contact, as well as the reported excess production of soluble factors (30).

It was also found that sublines continuously exposed to asbestos showed remarkably reduced expression of forkhead transcriptional factor 1 (FoxO1) (31). We reported that this reduced FoxO1 caused reduction of pro-apoptotic molecules such as Puma, Bim and Fas ligand in exposed sublines (CB1-3, CA1-3 and CR1), in addition to Bcl-2 overexpression induced by phosphorylation of signal tranducer and activator of transcription 3 (STAT3) caused by autocrine usage of overproduced IL-10 (31). FoxO1 is known to regulate various cell cycle regulators such as cyclins and cyclin-dependent kinase-inhibitors (CDK-Is) (32-36). Therefore, in this study we analyzed the expression of these cell cycle regulators and the status of cell cycle progression in sublines continuously exposed to asbestos, and compared the results with those obtained for Org cells.

\section{Materials and methods}

Cell lines and asbestos. Details of MT-2, Org and the asbestosinduced apoptosis resistant sublines (CA1-3, CB1-3 and CR1) have been reported previously $(22-24)$. These cells were maintained in a humidified atmosphere of $5 \% \mathrm{CO}_{2}$ at $37^{\circ} \mathrm{C}$ in RPMI-1640 medium supplemented with $10 \%$ fetal calf serum (FCS), streptomycin and penicillin. Seven asbestos-resistant sublines, CA1-3, CB1-3 and CR1, were generated by continuous exposure to $\mathrm{ChA}, \mathrm{ChB}$, and $\mathrm{CRD}$, respectively. As previously reported (22-24), the doses of asbestos fibers for continuous exposure were 5-10 $\mu \mathrm{g} / \mathrm{ml}$. These doses induced less than half of the cells to proceed to apoptosis when transiently exposed (22-24). The International Union Against Cancer standard ChA and ChB were kindly provided by the Department of Occupational Health at the National Institute for Occupational Health, South Africa. In addition, ChA, ChB and CRD were kindly provided as standard fibers from the Japan Association for the Study of Fiber Materials. The mineralogical features of fibers used have been reported previously (37).

Real-time RT-PCR. The expression levels of various cell cycle regulators such as CDK-Is including p21 ${ }^{\mathrm{Cip} 1}, \mathrm{p} 27^{\mathrm{Kip} 1}, \mathrm{p} 57^{\mathrm{Kip} 2}$, p16 ${ }^{\text {ink4a }}, \mathrm{p} 15^{\text {ink4b }}, \mathrm{p} 18^{\text {ink4c }}$, and p19 ${ }^{\text {ink4d }}$, and cyclins including cyclin A, B, D1, D2 and E, were analyzed in Org and continuously exposed sublines (CA1-3, CB1-3 and CR) using the real-time RT-PCR method. All the primers used are listed in Table I. Total cellular mRNA from ORG, CA1-3. CB1-3 and CR1 cells was extracted using the RNeasy Mini kit (Qiagen $\mathrm{GmbH}$, Hilden, Germany). After synthesis of the first strand of cDNA, real-time RT-PCR was performed using the SYBER Green method (Takara Bio Inc., Kusatsu, Japan) with the Mx3000P QPCR System (Agilent Technologies, Inc., Santa Clara, CA, USA) according to the manufacturer's instructions.

Western blotting. The procedures for western blotting were performed according to the previously reported methods (31). Briefly, MT-2Org cells and cells from asbestos continuously exposed sublines were lysed in $20 \mathrm{mM}$ Tris- $\mathrm{HCl}(\mathrm{pH} 7.5)$ containing $1 \mathrm{mM}$ EDTA, $1 \mathrm{mM}$ EGTA, $10 \mathrm{mM}$ 2-mercaptoethanol, $1 \%$ Triton $\mathrm{X}-100,1 \%$ sodium deoxycholate, $0.1 \%$ sodium lauryl sulfate, $150 \mathrm{mM} \mathrm{NaCl}$ and $1 \%$ protease 
Table I. Primers used for real-time RT-PCR.

Sequences

\begin{tabular}{lll}
\cline { 2 - 3 } Gene & \multicolumn{1}{c}{ Forward } & \multicolumn{1}{c}{ Reverse } \\
\hline GAPDH & 5'-GAGTCAACGGATTTGGTCGT -3' & 5'-TTGATTTTGGAGGGATCTCG-3' \\
Cyclin A & 5'-ATGTGTGCAGAAGGAGGTCC-3' & 5'-GAAGGTCCATGAGACAAGGC-3' \\
Cyclin B & 5'-CGAAGATCAACATGGCAGG-3' & 5'-CTTGGAGAGGCAGTATCAACC-3' \\
Cyclin D1 & 5'-ATGTGTGCAGAAGGAGGTCC-3' & 5'-CCTTCATCTTAGAGGCCACG-3' \\
Cyclin D2 & 5'-TGCAGAAGGACATCCAACC-3' & 5'-AGGAACATGCAGACAGCACC-3' \\
Cyclin E & 5'-TAAATGTCCCGCTCTGAGCC-3' & 5'-ACGTTTGCCTTCCTCTTCCT-3' \\
p21Cip1 & 5'-AGCAGAGGAAGACCATGTGG-3' & 5'-AGGCAGAAGATGTAGAGCGG-3' \\
p27Kip1 & 5'-AACGTGCGAGTGTCTAACGG-3' & 5'-CTTCCATGTCTCTGCAGTGC-3' \\
p57Kip2 & 5'-AGAGATCAGCGCCTGAGAAG-3' & 5'-TTGCTGCTACATGAACGGTC-3' \\
p16 $6^{\text {ink4a }}$ & 5'-ACCAGAGGCAGTAACCATGC-3' & 5'-CACATGAATGTGCGCTTAGG-3' \\
p15 $5^{\text {inkb }}$ & 5'-CGTTAAGTTTACGGCCAACG-3' & 5'-CATCATCATGACCTGGATCG-3' \\
p18 $8^{\text {ink4c }}$ & 5'-AGTTCCTGGTGAAGCACACG-3' & 5'-GGCTAACAACCTCATTCCTCC-3' \\
p19 ${ }^{\text {ink4d }}$ & 5'-ATGTCAACGTGCCTGATGG-3' & 5'-GGAGATCAGATTCAGCTGCC-3'
\end{tabular}

inhibitor cocktail (Sigma) and briefly sonicated. After centrifugation at $18,000 \mathrm{xg}$ for $10 \mathrm{~min}$, the supernatant was collected and measured using a BCA protein assay kit (Pierce, Rockford, IL, USA). Cell lysate containing $50 \mu \mathrm{g}$ protein was boiled in SDS-sample buffer and then subjected to SDS-PAGE separation. The resolved proteins were subsequently electrotransferred onto Immobilon P membranes (Millipore, Bedford, MA, USA). After initial blocking with Tris-buffered saline containing $0.2 \%$ Tween-20 (TBS-T) supplemented with $5 \% \mathrm{BSA}$ for $2 \mathrm{~h}$ membranes were then incubated with each primary antibody in TBS-T containing $1 \%$ BSA at dilutions recommended by the manufacturers for 1-2 $\mathrm{h}$ at room temperature (RT). Thereafter, the membrane was gently rinsed with TBS-T and then incubated with horseradish peroxidase-conjugated anti-mouse or anti-rabbit secondary antibodies in TBS-T at dilutions recommended by the manufacturers for $1 \mathrm{~h}$ at RT. After a final set of rinsing with TBS-T, the presence of the proteins of interest was evaluated using a chemiluminescence reaction mediated by an ECL Plus chemiluminescence detection kit (GE Healthcare, Little Chalfont, UK) and each was then visualized with Chemi-Stage (Toyobo, Osaka, Japan).

Western blotting for some of the cell cycle regulators was performed using mouse anti p21 $1^{\text {Cipl }}$ (F-5) (Santa Cruz Biotechnology), rabbit anti p16 ink4a $(\mathrm{C}-20)$ (Santa Cruz Biotechnology), rabbit anti-cyclin D1 (M-20) (Santa Cruz Biotechnology), and rabbit anti- $\beta$-actin as the control (Cell Signaling Technology, Danvers, MA, USA).

Flow cytometric analysis of the cell cycle. The individual cell cycle phases in logarithmically proliferating ORG, CA1-3, CB1-3 and CR1 cells were analyzed using flow cytometry. For the continuously exposed sublines CA1-3, CB1-3 and CR1, supplemented asbestos fibers were removed using density gradient centrifugation and cells without fibers were then cultured for one week before the analysis. All of these sublines and the Org MT-2 cells were cultured with bromodeoxyuridine (BrdU) for $30 \mathrm{~min}$, and after being washed twice with PBS the cells were incubated with fluorescence-labelled anti-BrdU antibody and 7-amino-actinomycin D (7AAD) for detection of DNA indices. The G1 phase in the cell cycle was then determined as BrdU-negative, DNA indices $=2 \mathrm{n}$ fraction, the S phase was BrdU-positive, and DNA indices $=2 \mathrm{n}<$ $<4$ fraction, while the $\mathrm{G} 2 / \mathrm{M}$ phases were BrdU-negative and DNA indices $=4 \mathrm{n}$ fraction.

Knock-down of FoxO1 in the MT-2Org cell line. Procedures regarding knock-down of FoxO1 in MT-2Org with lentivirus have been described (31). Lentivirus plasmid vectors pLKO.1-puro-Control having scramble shRNA sequence and pLKO.1-puro containing shRNA sequence targeting human FoxO1 (TRCN0000039579 and TRCN0000039580), and the packaging plasmids kit of pLP1, pLP2 and pLP/VSVG, were obtained from Sigma and Invitrogen (Carlsbad, CA, USA), respectively. Recombinant lentivirus was produced in HEK293T cells and MT-2Org cells were infected with recombinant lentivirus. Sublines having the shRNA expression cassette with puromycin resistance were established after culture with medium containing $1 \mu \mathrm{g} / \mathrm{ml}$ puromycin (Sigma) for 2 weeks. Resultant cell lines were designated Org-Ctrl (Scramble), Org-KD\#1 (TRCN0000039579) or Org-KD\#2 (TRCN0000039580). The amount of FoxO1 protein was determined with immunoblot analysis using anti-FoxO1 monoclonal antibody (Cell Signaling Technology).

\section{Results}

$m R N A$ expression of cell cycle regulators. As shown in Figs. 1 and 2, various cell cycle regulators in the cells of Org, CA1-3, CB1-3 and CR1 were analyzed. FoxO1 transcription factor is 

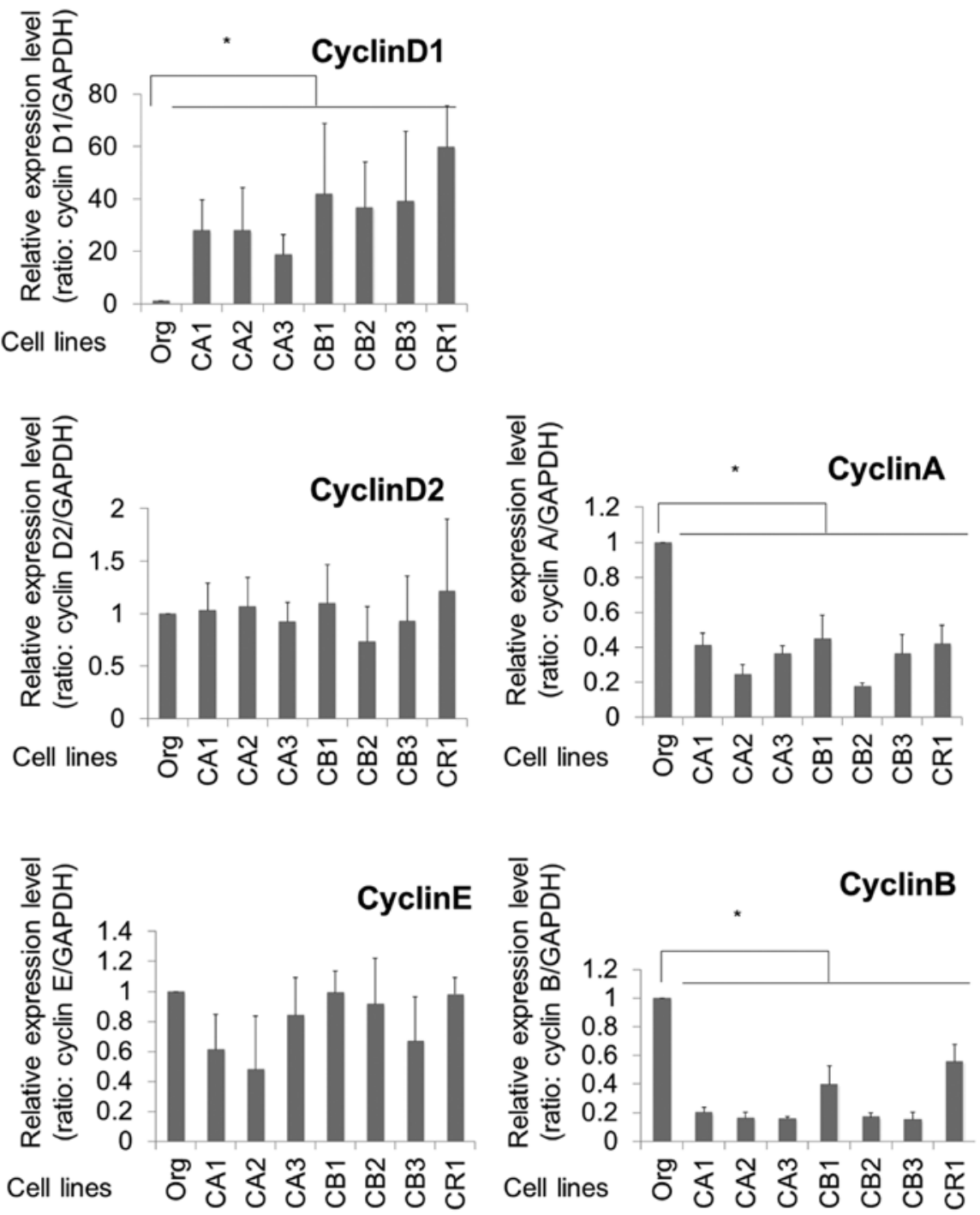

Figure 1. The gene expression of various cyclins in MT-2Org and sublines. The mRNA expression of cyclin D1, D2, E, A and B was analyzed using real-time RT-PCR in MT-2 Org and its sublines CA1-3, CB1-3 and CR1 continuously exposed to ChA, ChB and CRD, respectively. Graphs show mRNA expression level relative to MT-2 Org cells with a value of 1.0. Asterisk indicates a P-value (significance) $<0.05$.

known to negatively regulate cyclin D1 among the various cyclins. In addition, the sublines continuously exposed to asbestos (MT-2 Org, CA1-3, CB1-3 and CR1) showed remarkably reduced expression of the FoxO1 gene. As expected, cyclin D1 expression was remarkably enhanced in all the sublines by 20-60-fold when compared with that of Org cells. For other cyclins, expressions of cyclin D2 and E showed no differences between Org and sublines. Although cyclins A and $B$ showed decreased expression in sublines compared to Org cells, the reduction rate was $0.2-0.5$-fold in the sublines. The excess expression of cyclin D1 in the sublines was a remarkable finding among the cyclins examined.

In contrast to results for the cyclins, various CDK-Is exhibited reduced expression in sublines compared to MT-2 Org cells. As shown in Fig. 2, p21 $1^{\mathrm{Cip} 1}, \mathrm{p} 57^{\mathrm{Kip} 2}$, p18 ${ }^{\text {ink4c }}$ and 9 p $19^{\text {ink4d }}$ showed significantly reduced expression in the sublines.

Protein expression of cyclin D1 and CDK-Is. The representative protein expression of $\mathrm{p} 21^{\mathrm{Cip} 1}, \mathrm{p} 16^{\mathrm{ink} 4 \mathrm{a}}$ and cyclin D1 are shown in Fig. 3. Similar to findings for mRNA expression, cyclin D1 was expressed highly in sublines when compared to that in MT-2 Org cells. In addition, expressions of $\mathrm{p} 21^{\mathrm{Cip} 1}$ and $\mathrm{p} 16^{\text {ink4a }}$ were reduced in the sublines. Although $\mathrm{p} 16^{\text {ink4a }}$ mRNA was not representative because of the lower quality of real-time RT-PCR, it was clear that the expression of p16 $6^{\text {inkta }}$ decreased remarkably in sublines at the protein level.

Cell cycle phases in MT-2Org and sublines continuously exposed to asbestos fibers. As shown in Fig. 4A, cell cycle phases in MT-2 Org cells and the seven sublines continuously exposed to asbestos fibers (CA1-3, CB1-3 and CR1) were analyzed using staining with anti-BrdU antibody and DNA indices using 7AAD. The percentages of the $\mathrm{S}$ phase (cells with BrdU-positive and $2 n<<4 n$ in DNA indices) were then divided by the percentage of the G1 phase (cells with BrdU-negative and $2 \mathrm{n}$ in DNA indices). The results for all cell lines and MT-2 Org are shown in Fig. 4B, and S/G1 phase-population ratios are relative to a ratio of 1.0 for MT-2 Org. All sublines showed a higher ratio ranging from 2.6 to 4.3. This indicated that the cell cycle in sublines had progressed rapidly compared to that 

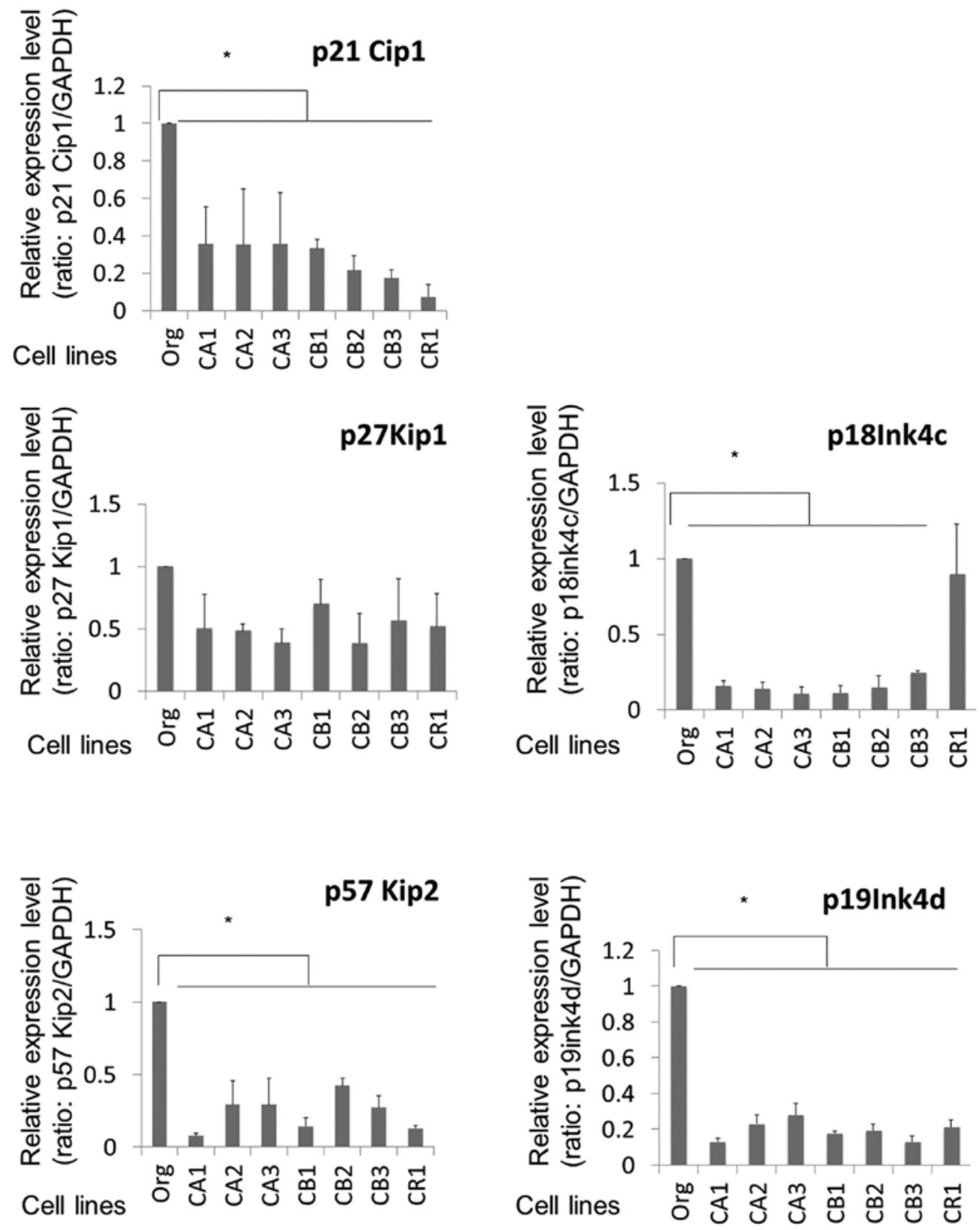

Figure 2. The gene expression of various cyclin-dependent kinase-inhibitors (CDK-Is) in MT-2Org and sublines. The mRNA expression of CDK-Is including $\mathrm{p} 21^{\text {Cip1 }}, \mathrm{p} 27^{\text {Kip1 }}, \mathrm{p} 57^{\text {Kip2 }}$, $18^{\text {ink4c }}$, and $\mathrm{p} 19^{\text {ink4d }}$ was analyzed using real-time RT-PCR in MT-2 Org and its sublines CA1-3, CB1-3 and CR1 continuously exposed to $\mathrm{ChA}, \mathrm{ChB}$ and CRD, respectively. Graphs show mRNA expression level relative to MT-2Org cells with a value of 1.0. Asterisk indicates a P-value (significance) $<0.05$.

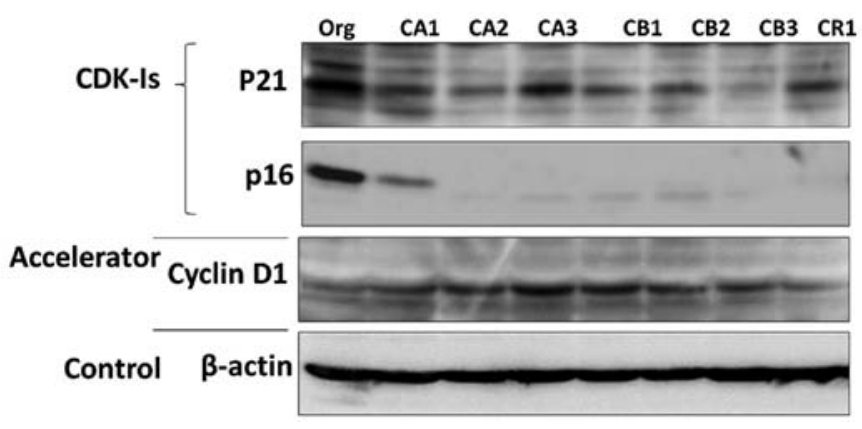

Figure 3. The protein expression of cell cycle regulators. Representative data regarding protein expression of $\mathrm{p} 21^{\mathrm{Cipl}}, \mathrm{p} 16^{\text {ink4a }}$ and cyclin D1 analyzed by western blotting for MT-2 Org and its sublines CA1-3, CB1-3 and CR1 continuously exposed to $\mathrm{ChA}, \mathrm{ChB}$ and $\mathrm{CRD}$, respectively. of MT-2 Org cells, as suggested from the data of cell cycle regulator expression, the remarkable excess expression of cyclin D1, and the reduced expression of CDK-Is.

Cyclin D1 expression in MT-2 Org knocked down forcibly by FoxO1. The sublines of MT-2 Org, which were continuously exposed to asbestos fibers, showed a remarkably decreased expression of FoxO1 as we reported previously. In addition, cyclin D1, which is negatively regulated by FoxO1, showed recovered expression in the sublines, and a similar pattern was observed for CDK-Is, which are positively regulated by FoxO1 and showed reduced expression in the sublines. To confirm the direct effects of reduced FoxO1 in MT-2 Org for the expression of cyclin D1, lentivirus-derived shRNA for FoxO1 was 

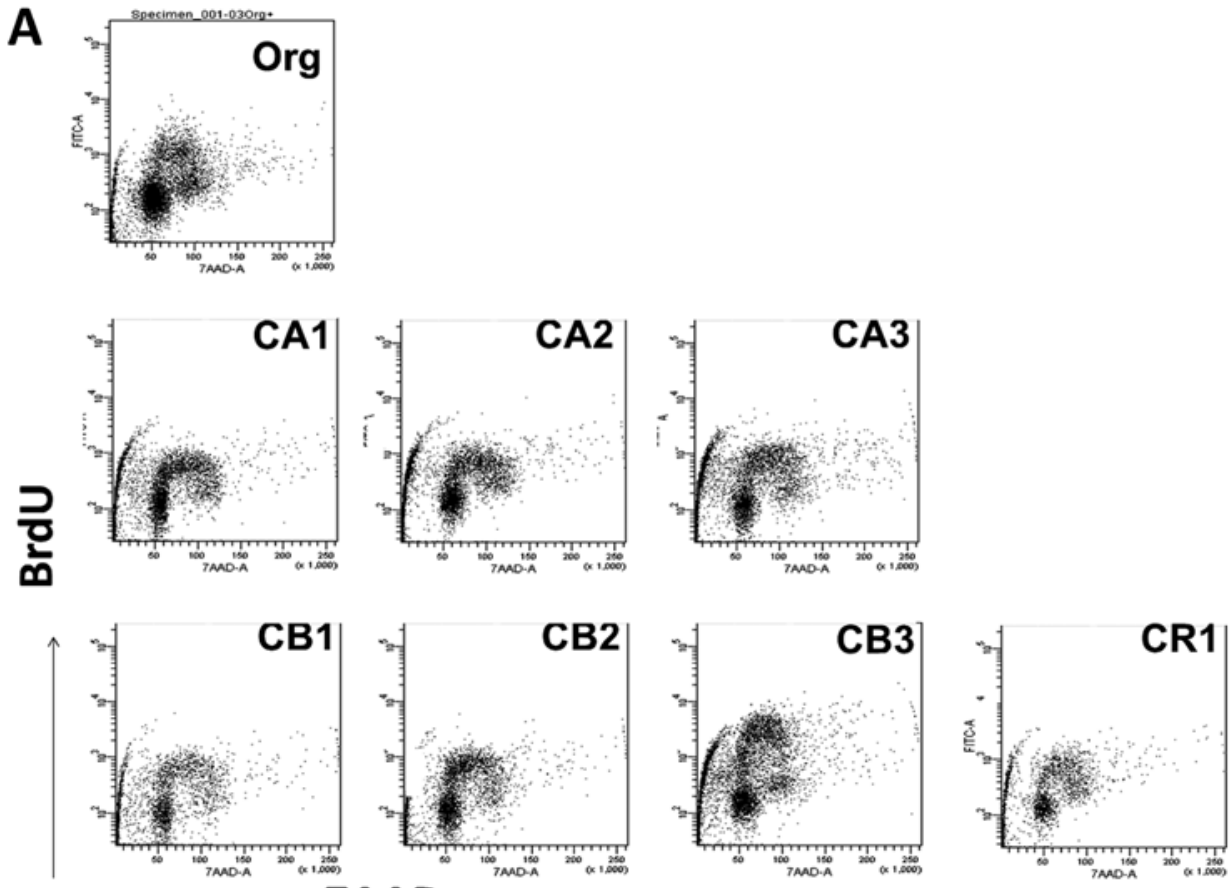

7AAD

B

\section{S phase/G1 phase}
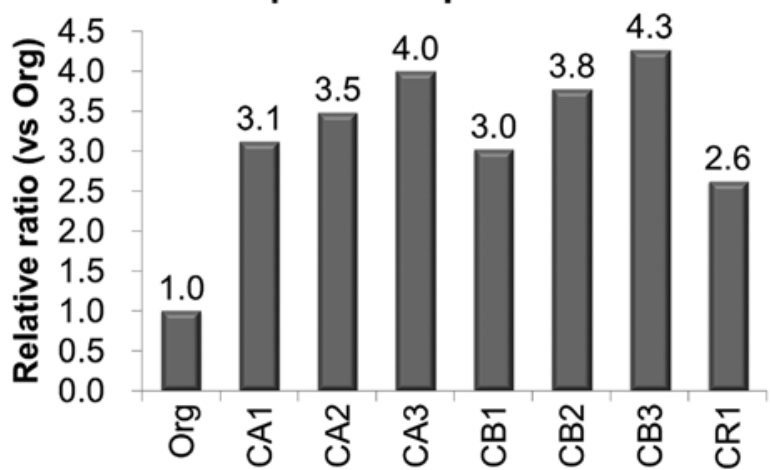

Figure 4. Cell cycle analyses. Cell cycle phases were analyzed using BrdU incorporated cells in MT-2 Org and its sublines CA1-3, CB1-3 and CR1 continuously exposed to $\mathrm{ChA}, \mathrm{ChB}$ and $\mathrm{CRD}$, respectively. (A) Representative flow cytometry findings for all cell lines examined. (B) The $\mathrm{S}$ phase population (percentage) divided by the G1 phase population (percentage) in individual cell lines including MT-2 Org, CA1-3, CB1-3 and CR, with ratios being relative to a value of 1.0 for the MT-2 Org line.

induced in MT-2Org, and the expression of cyclin D1 in MT-2 Org and the knocked-down subline was analyzed. As shown in Fig. 5A, two of the knocked-down cells (KD\#1 and KD\#2) showed reduced expression of FoxO1. Additionally, the mRNA expression of cyclin D1 was assayed using KD\#1 cells and scrambled sequence transfected cells. Although the expression level in KD\#1 was not enhanced remarkably, as we observed in sublines continuously exposed to asbestos fibers for more than one year, the expression of cyclin D1 was significantly upregulated in KD\#1 compared to that in scrambled transfected cells.

\section{Discussion}

Asbestos-exposed patients show complications comprising malignant tumors such as lung cancer and malignant mesothelioma (4-6). In addition, these individuals might have an increased risk of developing other tumors such as laryngeal, gastrointestinal and bladder cancers $(38,39)$. Pulmonary regions are also affected by carcinogenic activities such as ROS production, physical impairment of cellular DNA due to the mineralogical features of the asbestos fibers, and absorption of other carcinogenic substances inhaled into the lung, all of which may lead to the development of malignant tumors. However, a consideration of other cancers and the long latent period (30-50 years) for pulmonary and pleural occurrence of cancers suggest that reduction of antitumor immunity may be an important factor in the development of asbestos-induced tumors (4-6). It is from this viewpoint that we have been investigating the immunological effects of asbestos fibers on immune competent cells. Our results showed that NK cells exhibited reduced killing activity and expression of various activating receptors, as well as a decrease of MAPK signaling in experiments involving an asbestos-exposed human NK cell line, freshly isolated NK cells exposed to asbestos in vitro, and 


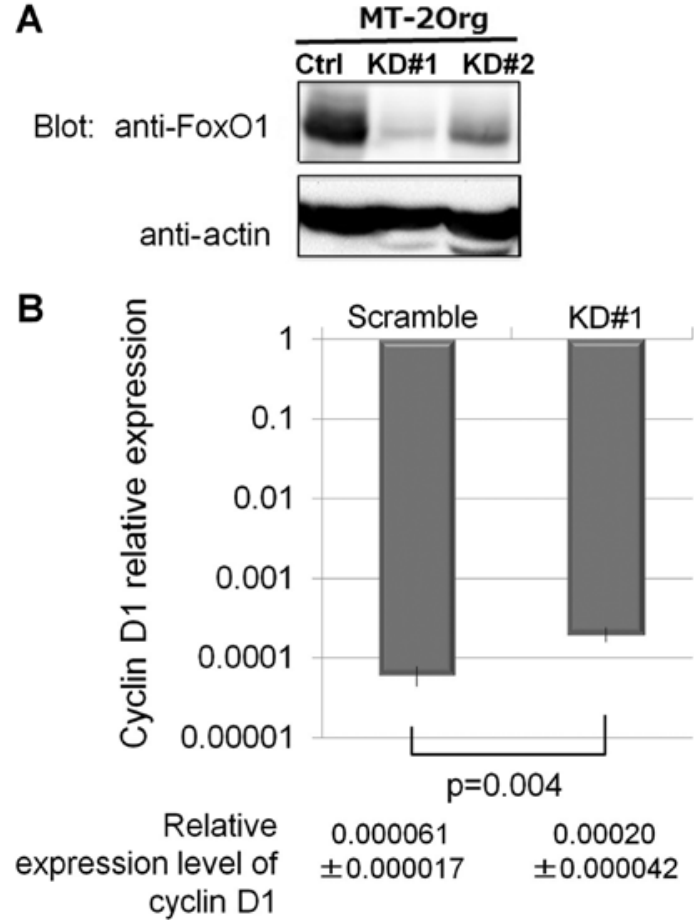

Figure 5. The effects of FoxO1 knock-down on the expression of cyclin D1 (A) Artificial knock-down of the FoxO1 gene in the MT-2 Org line was performed using lentivirus-mediated shRNA transfection. The reduction of FoxO1 protein in two clones (KD\#1 and KD\#2) is shown via immunoblotting. (B) Cyclin D1 mRNA expression assayed using real-time RT-PCR in MT-2 Org and the KD\#1 clone silenced by shRNA for FoxO1. The relative expression level of cyclin D1 was enhanced significantly in KD\#1 cells.

\section{Enhancement of Treg function}
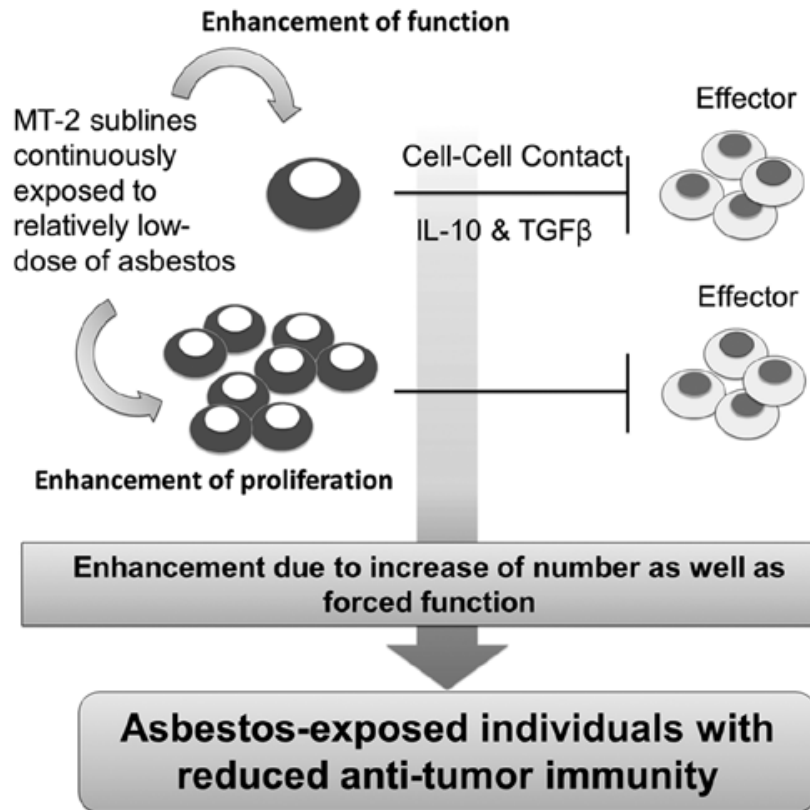

Figure 6. Schematic summary of the effects on Treg following continuous exposure to asbestos fibers, the 'enhancement of Treg function'. The function of Treg was enhanced through cell-cell contact and excess production of typical soluble factors such as IL-10 and TGF- $\beta$. In addition to these functional enhancements, the volume of Treg may increase following asbestos exposure through the remarkable reduction of the FoxO1 transcription factor. Both effects of asbestos exposure on Treg reduces antitumor immunity in asbestos-exposed individuals. in NK cells derived from asbestos-exposed patients with PP or MM (17,18). CTLs showed reduced differentiation and clonal expansion when $\mathrm{CD}^{+} \mathrm{T}$ cells were put into the MLR assay with reduction of cell attacking molecules such as granzyme $B$ and perforin $(19,20)$. Investigation of $\mathrm{T}$ helper cells showed that CXCR3 expression and IFN- $\gamma$ production were reduced in trials involving the cell line model, freshly isolated cells from HD and continuously exposed to asbestos in vitro, and in $\mathrm{CD}^{+}$cells derived from patients with PP or MM $(15,26,27)$.

The other immune cell that plays an important role in antitumor immunity is Treg. If the function and volume of Treg are enhanced, the activity of tumor-killing T cells is suppressed, particularly in the area surrounding tumor cells, and antitumor immunity is subsequently reduced (40-42). To investigate the possibility of enhanced Treg function following its continuous exposure to asbestos, we investigated Treg function using MT-2 cells, which were reported to possess Treg function $(29,30)$. Our findings indicated that MT-2 cells exposed continuously to asbestos with a relatively low dose did not exhibit apoptosis when exposed transiently, showed enhancement of Treg function via cell-cell contact, and revealed an increase of soluble factors to function as Treg, namely, IL-10 and TGF- $\beta$, as we reported previously $(22,24,30)$.

The next goal was to determine how asbestos alters cell proliferation or cell cycle progression in Treg. A key fact for this investigation was the remarkably reduced expression of FoxO1 transcription factor found in MT-2 sublines exposed continuously to asbestos fibers such as $\mathrm{ChA}, \mathrm{ChB}$, and CRD. These sublines showed resistance against the asbestos-induced apoptosis via upregulation of IL-10, increased phosphorylation of STAT3 by autocrine usage of IL-10, and subsequent upregulation of Bcl-2 located downstream of STAT3 (22). Moreover, our recent study revealed that reduced FoxO1 caused reduction of other apoptosis-related molecules such as Bim, Puma, and Fas ligand, which are known to be regulated by FoxO1 (31).

FoxO1 is known to regulate various cell cycle regulators, such as cyclin D1 and CDK-Is. Since cyclin D1 is negatively regulated and CDK-Is are positively regulated, it seems that FoxO1 control of the cell cycle does not proceed quickly. However, if continuous asbestos exposure causes massive downregulation of FoxO1, the cell proliferating activity might be accelerated in such cells. Therefore, the expression of cell cycle regulators in MT-2 Org and its sublines was examined. Results showed an overall tendency toward acceleration of cell cycle progression, since cyclin D1 was highly expressed, CDK-Is showed reduced expression, and the $\mathrm{S}$ phase in sublines increased in comparison to MT-2 Org cells. The increased expression of cyclin D1 was regulated by FoxO1, since artificially silenced cells from MT-2 Org showed increased cyclin D1 expression.

A consideration of the overall results and our previous findings reveals that MT-2 sublines continuously exposed to asbestos fibers such as $\mathrm{ChA}, \mathrm{ChB}$ and $\mathrm{CRD}$ showed enhanced Treg function via cell-cell contact and excess secretion of typical two soluble factors, namely, IL-10 and TGF- $\beta$, and examination of Treg in the asbestos-exposed population may reflect enhancement of function and proliferation, quality and volume (Fig. 6). However, it is difficult to examine these facts in asbestos-exposed patients because the function and volume of Treg needs to be analyzed in the tumor-surrounding area 
instead of peripheral blood. This evaluation therefore needs time. Notwithstanding these considerations, our cell line model clearly showed that asbestos causes a reduction of antitumor immunity in asbestos-exposed individuals and makes them more sensitive to the development of tumors after a long-term latent period following the initial exposure to asbestos.

Future studies are needed to evaluate Treg function and volume in the surrounding areas of asbestos-induced tumors, and to develop preventive procedures to neutralize asbestosinduced enhancement of Treg through the use of natural products in foods or plants. These approaches may be helpful for chemoprevention of asbestos-induced tumorigenesis.

\section{Acknowledgements}

This study was supported by the Private University Strategic Research Base Formation Support Project (2011 to 2016), Kakenhi 15K08788, the Japanese Society for the Promotion of Science, and Research Grants from Kawasaki Medical School (27B058, 26B53, 25B67, 24S4 and 23S6).

\section{References}

1. Parkes WR: Asbestos-related disorders. Br J Dis Chest 67: 261-300, 1973 .

2. Mossman BT and Gee JB: Asbestos-related diseases. N Engl J Med 320: 1721-1730, 1989.

3. Peacock C, Copley SJ and Hansell DM: Asbestos-related benign pleural disease. Clin Radiol 55: 422-432, 2000.

4. Morinaga K, Kishimoto T, Sakatani M, Akira M, Yokoyama K and Sera Y: Asbestos-related lung cancer and mesothelioma in Japan. Ind Health 39: 65-74, 2001.

5. O'Reilly KM, Mclaughlin AM, Beckett WS and Sime PJ: Asbestos-related lung disease. Am Fam Physician 75: 683-688, 2007.

6. Lazarus A, Massoumi A, Hostler J and Hostler DC: Asbestosrelated pleuropulmonary diseases: Benign and malignant Postgrad Med 124: 116-130, 2012.

7. Kamp DW, Graceffa P, Pryor WA and Weitzman SA: The role of free radicals in asbestos-induced diseases. Free Radic Biol Med 12: 293-315, 1992.

8. Shukla A, Gulumian M, Hei TK, Kamp D, Rahman Q and Mossman BT: Multiple roles of oxidants in the pathogenesis of asbestos-induced diseases. Free Radic Biol Med 34: 1117-1129, 2003

9. Toyokuni S: Mechanisms of asbestos-induced carcinogenesis. Nagoya J Med Sci 71: 1-10, 2009.

10. Toyokuni S: Role of iron in carcinogenesis: Cancer as a ferrotoxic disease. Cancer Sci 100: 9-16, 2009.

11. Lemen RA, Dement JM and Wagoner JK: Epidemiology of asbestos-related diseases. Environ Health Perspect 34: 1-11, 1980.

12. Lee S, Matsuzaki H, Kumagai-Takei N, Yoshitome K, Maeda M, Chen Y, Kusaka M, Urakami K, Hayashi H, Fujimoto W, et al: Silica exposure and altered regulation of autoimmunity. Environ Health Prev Med 19: 322-329, 2014

13. Hayashi H, Miura Y, Maeda M, Murakami S, Kumagai N, Nishimura Y, Kusaka M, Urakami K, Fujimoto W and Otsuki T: Reductive alteration of the regulatory function of the CD4(+) CD25(+) T cell fraction in silicosis patients. Int J Immunopathol Pharmacol 23: 1099-1109, 2010.

14. Lee S, Hayashi H, Maeda M, Chen Y, Matsuzaki H, TakeiKumagai N, Nishimura Y, Fujimoto W and Otsuki T: Environmental factors producing autoimmune dysregulation - chronic activation of T cells caused by silica exposure. Immunobiology 217: 743-748, 2012.

15. Otsuki T, Matsuzaki H, Lee S, Kumagai-Takei N, Yamamoto S, Hatayama T, Yoshitome K and Nishimura Y: Environmental factors and human health: Fibrous and particulate substanceinduced immunological disorders and construction of a health-promoting living environment. Environ Health Prev Med 21: 71-81, 2016.
16. Kumagai-Takei N, Maeda M, Chen Y, Matsuzaki H, Lee S, Nishimura Y, Hiratsuka J and Otsuki T: Asbestos induces reduction of tumor immunity. Clin Dev Immunol 2011: 481439, 2011.

17. Nishimura Y, Miura Y, Maeda M, Kumagai N, Murakami S, Hayashi H, Fukuoka K, Nakano T and Otsuki T: Impairment in cytotoxicity and expression of NK cell- activating receptors on human NK cells following exposure to asbestos fibers. Int J Immunopathol Pharmacol 22: 579-590, 2009.

18. Nishimura Y, Maeda M, Kumagai N, Hayashi H, Miura Y and Otsuki T: Decrease in phosphorylation of ERK following decreased expression of NK cell-activating receptors in human NK cell line exposed to asbestos. Int J Immunopathol Pharmacol 22: 879-888, 2009.

19. Kumagai-Takei N, Nishimura Y, Maeda M, Hayashi H, Matsuzaki H, Lee S, Hiratsuka J and Otsuki T: Effect of asbestos exposure on differentiation of cytotoxic T lymphocytes in mixed lymphocyte reaction of human peripheral blood mononuclear cells. Am J Respir Cell Mol Biol 49: 28-36, 2013.

20. Kumagai-Takei N, Nishimura Y, Maeda M, Hayashi H, Matsuzaki H, Lee S, Kishimoto T, Fukuoka K, Nakano T and Otsuki T: Functional properties of CD8 (+) lymphocytes in patients with pleural plaque and malignant mesothelioma. J Immunol Res 2014: 670140, 2014.

21. Hyodoh F, Takata-Tomokuni A, Miura Y, Sakaguchi H, Hatayama T, Hatada S, Katsuyama H, Matsuo Y and Otsuki T: Inhibitory effects of anti-oxidants on apoptosis of a human polyclonal T-cell line, MT-2, induced by an asbestos, chrysotile-A. Scand J Immunol 61: 442-448, 2005.

22. Miura Y, Nishimura Y, Katsuyama H, Maeda M, Hayashi H, Dong M, Hyodoh F, Tomita M, Matsuo Y, Uesaka A, et al: Involvement of IL-10 and Bcl-2 in resistance against an asbestosinduced apoptosis of T cells. Apoptosis 11: 1825-1835, 2006.

23. Maeda M, Yamamoto S, Chen Y, Kumagai-Takei N, Hayashi H, Matsuzaki H, Lee S, Hatayama T, Miyahara N, Katoh M, et al: Resistance to asbestos-induced apoptosis with continuous exposure to crocidolite on a human T cell. Sci Total Environ 429: 174-182, 2012.

24. Maeda M, Chen Y, Hayashi H, Kumagai-Takei N, Matsuzaki H, Lee S, Nishimura Y and Otsuki T: Chronic exposure to asbestos enhances TGF- $\beta 1$ production in the human adult T cell leukemia virus-immortalized T cell line MT-2. Int J Oncol 45: 2522-2532, 2014.

25. Maeda M, Chen Y, Kumagai-Takei N, Hayashi H, Matsuzaki H, Lee S, Hiratsuka J, Nishimura Y, Kimura Y and Otsuki T: Alteration of cytoskeletal molecules in a human $\mathrm{T}$ cell line caused by continuous exposure to chrysotile asbestos. 218: 11841191, 2013.

26. Maeda M, Nishimura Y, Hayashi H, Kumagai N, Chen Y, Murakami S, Miura Y, Hiratsuka J, Kishimoto T and Otsuki T: Reduction of CXC chemokine receptor 3 in an in vitro model of continuous exposure to asbestos in a human T-cell line, MT-2. Am J Respir Cell Mol Biol 45: 470-479, 2011.

27. Maeda M, Nishimura Y, Hayashi H, Kumagai N, Chen Y, Murakami S, Miura Y, Hiratsuka J, Kishimoto T and Otsuki T: Decreased CXCR3 expression in $\mathrm{CD}^{+} \mathrm{T}$ cells exposed to asbestos or derived from asbestos-exposed patients. Am J Respir Cell Mol Biol 45: 795-803, 2011.

28. Matsuzaki H, Maeda M, Lee S, Nishimura Y, Kumagai-Takei N, Hayashi H, Yamamoto S, Hatayama T, Kojima Y, Tabata R, et al: Asbestos-induced cellular and molecular alteration of immunocompetent cells and their relationship with chronic inflammation and carcinogenesis. J Biomed Biotechnol 2012: 492608, 2012.

29. Hamano R, Wu X, Wang Y, Oppenheim JJ and Chen X: Characterization of MT-2 cells as a human regulatory T cell-like cell line. Cell Mol Immunol 12: 780-782, 2015.

30. Ying C, Maeda M, Nishimura Y, Kumagai-Takei N, Hayashi H, Matsuzaki H, Lee S, Yoshitome K, Yamamoto S, Hatayama T, et al: Enhancement of regulatory $\mathrm{T}$ cell-like suppressive function in MT-2 by long-term and low-dose exposure to asbestos. Toxicology 338: 86-94, 2015.

31. Matsuzaki H, Lee S, Maeda M, Kumagai-Takei N, Nishimura Y and Otsuki T: FoxO1 regulates apoptosis induced by asbestos in the MT-2 human T-cell line. J Immunotoxicol 13: 620-627, 2016.

32. Arden KC: FoxO: Linking new signaling pathways. Mol Cell 14: 416-418, 2004

33. Accili D and Arden KC: FoxOs at the crossroads of cellular metabolism, differentiation, and transformation. Cell 117: 421-426, 2004 
34. Huang $\mathrm{H}$ and Tindall DJ: CDK2 and FOXO1: A fork in the road for cell fate decisions. Cell Cycle 6: 902-906, 2007.

35. Liu P, Kao TP and Huang H: CDK1 promotes cell proliferation and survival via phosphorylation and inhibition of FOXO1 transcription factor. Oncogene 27: 4733-4744, 2008.

36. Eijkelenboom A and Burgering BM: FOXOs: Signalling integrators for homeostasis maintenance. Nat Rev Mol Cell Biol 14: 83-97, 2013.

37. Kohyama N, Shinohara Y and Suzuki Y: Mineral phases and some reexamined characteristics of the International Union Against Cancer standard asbestos samples. Am J Ind Med 30: 515-528, 1996.

38. Gao FF and Oury TD: Other neoplasia. In: Pathology of AsbestosAssociated Diseases. Roggi VL, Oury TD and Sporn TA (eds.) 3rd edit. Springer, Berlin, pp177-192, 2014.
39. Craighead JE: Nonthoracic cancers possibly resulting from asbestos exposure. In: Asbestos and its Diseases. Craighead JE and Gibbs AR (eds). Oxford University Press, New York, NY, pp230-252, 2008.

40. Sakaguchi S, Ono M, Setoguchi R, Yagi H, Hori S, Fehervari Z, Shimizu J, Takahashi T and Nomura T: Foxp $3^{+} \mathrm{CD} 25^{+} \mathrm{CD} 4^{+}$ natural regulatory $\mathrm{T}$ cells in dominant self-tolerance and autoimmune disease. Immunol Rev 212: 8-27, 2006.

41. Nishikawa $\mathrm{H}$ and Sakaguchi S: Regulatory $\mathrm{T}$ cells in tumor immunity. Int J Cancer 127: 759-767, 2010.

42. Yamaguchi $\mathrm{T}$ and Sakaguchi S: Regulatory $\mathrm{T}$ cells in immune surveillance and treatment of cancer. Semin Cancer Biol 16: 115-123, 2006. 